\title{
On bivariate fundamental polynomials
}

\author{
V. Vardanyan (vahagn.vardanyan94@gmail.com) \\ Department of Mathematics and Mechanics \\ Yerevan State University \\ A. Manukyan St. 1 \\ 0025 Yerevan, Armenia
}

\begin{abstract}
An $n$-independent set in two dimensions is a set of nodes admitting (not necessarily unique) bivariate interpolation with polynomials of total degree at most $n$. For an arbitrary $n$-independent node set $\mathcal{X}$ we are interested with the property that each node possesses an $n$-fundamental polynomial in form of product of linear or quadratic factors. In the present paper we show that each node of $\mathcal{X}$ has an $n$ fundamental polynomial, which is a product of lines, if $\# \mathcal{X} \leq 2 n+1$. Next we prove that each node of $\mathcal{X}$ has an $n$-fundamental polynomial, which is a product of lines or conics, if $\# \mathcal{X} \leq 2 n+[n / 2]+1$. We have a counterexample in each case to show that the results are not valid in general if $\# \mathcal{X} \geq 2 n+2$ and $\# \mathcal{X} \geq 2 n+[n / 2]+2$, respectively.
\end{abstract}

Key words: Bivariate polynomial, interpolation, fundamental polynomial, conic, $n$-poised, $n$-independent nodes.

Mathematics Subject Classification (2010):

primary: 41A05, 41A63; secondary 14H50.

\section{Introduction}

Let $\Pi_{n}$ be the space of bivariate polynomials of total degree at most $n$ :

$$
\Pi_{n}=\left\{\sum_{i+j \leq n} a_{i j} x^{i} y^{j}: a_{i j} \in \mathbb{R}\right\} .
$$

We have that

$$
N:=N_{n}:=\operatorname{dim} \Pi_{n}=\left(\begin{array}{c}
n+2 \\
2
\end{array}\right)
$$


Consider a set of distinct nodes (points)

$$
\mathcal{X}_{s}=\left\{\left(x_{1}, y_{1}\right),\left(x_{2}, y_{2}\right), \ldots,\left(x_{s}, y_{s}\right)\right\} .
$$

The problem of finding a polynomial $p \in \Pi_{n}$ which satisfies the conditions

$$
p\left(x_{i}, y_{i}\right)=c_{i}, \quad i=1,2, \ldots s,
$$

is called interpolation problem. A polynomial $p \in \Pi_{n}$ is called an $n$-fundamental polynomial for a node $A=\left(x_{k}, y_{k}\right) \in \mathcal{X}_{s}$ if

$$
p\left(x_{i}, y_{i}\right)=\delta_{i k}, \quad i=1, \ldots, s,
$$

where $\delta$ is the Kronecker symbol. We denote this fundamental polynomial by $p_{k}^{\star}=p_{A}^{\star}=p_{A, \mathcal{X}_{s}}^{\star}$. Sometimes we call fundamental also a polynomial that vanishes at all nodes of $\mathcal{X}$ but one, since it is a nonzero constant times a fundamental polynomial.

Definition 1.1. A set of nodes $\mathcal{X}$ is called $n$-independent if all its nodes have fundamental polynomials. Otherwise, $\mathcal{X}$ is called $n$-dependent.

Fundamental polynomials are linearly independent. Therefore a necessary condition of $n$-independence is $\# \mathcal{X} \leq N$. Having fundamental polynomials of all nodes of $\mathcal{X}$ we get a solution of general interpolation problem (1.1) by using the Lagrange formula:

$$
p(x, y)=\sum_{i=1}^{s} c_{i} p_{i}^{\star}(x, y) .
$$

Thus we get readily that the node set $\mathcal{X}_{s}$ is $n$-independent if and only if it is $n$-solvable, meaning that for any data $\left\{c_{1}, \ldots, c_{s}\right\}$ there exists a (not necessarily unique) polynomial $p \in \Pi_{n}$ satisfying the conditions (1.1).

Definition 1.2. The interpolation problem with the set of nodes $\mathcal{X}_{s}$ is called $n$-poised if for any data $\left\{c_{1}, \ldots, c_{s}\right\}$ there exists a unique polynomial $p \in \Pi_{n}$, satisfying the conditions (1.1).

A necessary condition for $n$-poisedness is $s=\# \mathcal{X}_{s}=N$. We have also that a set $\mathcal{X}_{N}$ is $n$-poised if and only if it is $n$-independent. The following proposition is based on an elementary Linear Algebra argument.

Proposition 1.3. The interpolation problem with the set of nodes $\mathcal{X}_{N}$ is $n$-poised if and only if the following condition holds:

$$
p \in \Pi_{n}, p\left(x_{i}, y_{i}\right)=0, i=1, \ldots, N \Rightarrow p=0 .
$$


Now let us bring some results on $n$-independence we shall use in the sequel. Let us start with the following simple but important result of Severi (see [5]):

Theorem $1.4([5])$. Any set $\mathcal{X}$, with $\# \mathcal{X} \leq n+1$, is n-independent.

Remark 1.5. For each node $A \in \mathcal{X}$ here we can find $n$-fundamental polynomial which is a product of $\# \mathcal{X}-1 \leq n$ lines, each of which passes through a respective node of $\mathcal{X} \backslash\{A\}$ and does not pass through $A$.

Next two results extend the Severi theorem to the cases of sets with no more than $2 n+1$ (see [1], Proposition 1) and $3 n-1$ (see [3], Theorem 5.3) nodes, respectively.

Theorem 1.6 ([1]). Any set $\mathcal{X}$, with $\# \mathcal{X} \leq 2 n+1$, is $n$-independent, if and only if no $n+2$ nodes of $\mathcal{X}$ are collinear.

Theorem $1.7([3])$. Let $\mathcal{X}$ be set of nodes with $\# \mathcal{X} \leq 3 n$. Then the set $\mathcal{X}$ is $n$-dependent if and only if one of the following hold:

i) $n+2$ nodes of $\mathcal{X}$ are collinear,

ii) $2 n+2$ nodes of $\mathcal{X}$ are lying on a conic,

iii) $\# \mathcal{X}=3$, there are curves $\gamma \in \Pi_{3}$ and $p \in \Pi_{n}$ such that $\gamma \cap p=\mathcal{X}$.

Here we use the same letter, say $p$, to denote the polynomial $p \in \Pi_{n} \backslash \Pi_{0}$ and the algebraic curve defined by the equation $p(x, y)=0$. We denote lines and conics by $\alpha$ and $\beta$, respectively.

Note that, according to Theorem 1.3, the interpolation problem with $\mathcal{X}_{N}$ is $n$-poised if and only if there is no algebraic curve of degree $\leq n$ passing through all the nodes of $\mathcal{X}_{N}$.

At the end of this section let us discuss the problem we consider. In view of the Lagrange formula (1.2) it is very important to find $n$-independent (i.e., $n$-solvable) sets for which the fundamental polynomials have the simplest possible forms. In Section 2 we characterize $n$-independent sets for which all fundamental polynomials are products of lines. It is worth mentioning that for the natural lattice, introduced by Chung and Yao in [2], the fundamental polynomials have the mentioned forms. But in this case the nodes satisfy very special conditions. Namely, they are intersection points of some $n+2$ given lines. In our characterization (see forthcoming Theorem 2.1, Proposition 2.2) the restrictions on the node set are much more weak. In Sections 3 we consider a much more involved problem. Here we characterize $n$-independent node sets for which all fundamental polynomials are products of lines or conics. 


\section{The fundamental polynomials as products of lines}

Theorem 2.1. Let $\mathcal{X}$ be an $n$-independent set of nodes with $\# \mathcal{X} \leq 2 n+1$. Then for each node of $\mathcal{X}$ there is an $n$-fundamental polynomial, which is a product of lines. Moreover, this statement is not true in general for $n$ independent node sets $\mathcal{X}$ with $\# \mathcal{X} \geq 2 n+2$ and $n \geq 2$.

The first statement of Theorem follows from the following result which covers more wider setting.

Proposition 2.2. Let $\mathcal{X}$ be a set of nodes with $\# \mathcal{X} \leq 2 n+1$ and $A \in \mathcal{X}$. Then the following three statements are equivalent

i) The node $A$ has an $n$-fundamental polynomial,

ii) The node $A$ has an $n$-fundamental polynomial, which is a product of linear factors,

iii) No $n+1$ nodes of $\mathcal{X} \backslash\{A\}$ are collinear together with the node $A$.

\section{The fundamental polynomials as products of lines and conics}

Theorem 3.1. Let $\mathcal{X}$ be an $n$-independent set of nodes with $\# \mathcal{X} \leq 2 n+$ $[n / 2]+1$. Then for each node of $\mathcal{X}$ there is an $n$-fundamental polynomial, which is a product of lines and conics. Moreover, this statement is not true in general for $n$-independent node sets $\mathcal{X}$ with $\# \mathcal{X} \geq 2 n+[n / 2]+2$ and $n \geq 3$.

The first statement of Theorem follows from the following result which covers more wider setting.

Proposition 3.2. Let $\mathcal{X}$ be a set of nodes with $\# \mathcal{X} \leq 2 n+[n / 2]+1$ and $A \in \mathcal{X}$. Then the following three statements are equivalent:

i) The node $A$ has an n-fundamental polynomial,

ii) The node $A$ has an $n$-fundamental polynomial, which is a product of lines and conics,

iii) a) no $n+1$ nodes of $\mathcal{X} \backslash\{A\}$ are collinear together with $A$,

b) if $n+1$ nodes of $\mathcal{X} \backslash\{A\}$ are collinear and are lying in a line $\alpha$ then no $n$ nodes of $\mathcal{X} \backslash(A \cup \alpha)$ are collinear together with $A$,

c) no $2 n+1$ nodes of $\mathcal{X} \backslash\{A\}$ are lying on an irreducible conic together with A. 


\section{References}

[1] D. Eisenbud, M. Green and J. Harris (1996)

Cayley-Bacharach theorems and conjectures, Bull. Amer. Math. Soc. (N.S.), 33(3), 295-324.

[2] Chung, K. C. and Yao, T. H., On lattices admitting unique Lagrange interpolation, SIAM J. Numer. Anal. 14 (1977), 735-743.

[3] H. Hakopian and A. Malinyan, Characterization of $n$-independent sets with no more than 3n points, Jaén J. Approx. 4(2012), 119 - 134.

[4] J. Radon, Zur mechanischen Kubatur, Monatsh. Math. 52 (1948) 286300.

[5] F. Severi, Vorlesungen Ëuber Algebraische Geometrie (Teubner, Berlin, 1921). 